\title{
PAINEL-Oncologia: uma Ferramenta de Gestão
}

doi: https://doi.org/10.32635/2176-9745.RBC.2020v66n2.827

\author{
Oncology-PANEL: Tool for Management \\ PANEL-Oncología: una Herramienta de Gestión
}

\section{Adriana Tavares de Moraes Atty ${ }^{1}$; Beatriz Cordeiro Jardim²; Maria Beatriz Kneipp Dias ${ }^{3}$; Arn Migowski; Jeane Glaucia Tomazelli ${ }^{5}$}

Resumo

Introduçáo: A necessidade de monitorar o intervalo entre o diagnóstico de uma neoplasia maligna e o início do tratamento oncológico no Sistema Público de Saúde demandou a construção de uma nova ferramenta. Para tanto, foi desenvolvido o PAINEL-Oncologia. Objetivo: Apresentar o processo de elaboração da ferramenta, suas aplicaçóes, potencialidades e limites, apontando ainda perspectivas futuras. Método: O painel foi construído a partir do relacionamento determinístico de informaçóes diagnósticas e de tratamento obtidas no Sistema de Informação Ambulatorial, por meio do Boletim de Produção Ambulatorial Individualizado e da Autorização de Procedimento de Alta Complexidade, no Sistema de Informação Hospitalar e no Sistema de Informaçóes de Câncer, utilizando-se o Cartão Nacional de Saúde e o diagnóstico de neoplasia como chave identificadora do caso. Resultados: A ferramenta disponibiliza três painéis: 1 - Painel: Monitoramento do início do tratamento oncológico, que exclui os cânceres de pele náo melanoma e tireoide; 2 - Painel: Casos sem data de diagnóstico por ano de tratamento; e 3 - Painel: Casos diagnosticados de câncer de pele náo melanoma e tireoide; e um conjunto de gráficos e tabelas com filtros para seleção. Conclusáo: O PAINEL-Oncologia foi disponibilizado aos gestores e a órgãos de controle em 15 de maio de 2019. Trata-se de uma ferramenta ágil e acessível ao gestor para o monitoramento do tempo do primeiro tratamento do câncer. Contudo, as informaçôes apresentadas no PAINEL-Oncologia dependem do registro e da qualidade das informaçôes dos Sistemas de Informação em Saúde dos quais ele consome informaçôes.

Palavras-chave: Sistema de Informação em Saúde; Oncologia; Monitoramento; Gestão da Informação em Saúde; Registro Médico Coordenado.

\begin{abstract}
Introduction: It was necessary to construct a new tool to monitor the interval between the diagnosis of a malignant neoplasm and the beginning of cancer treatment in the Public Health System. For this purpose, the Oncology-PANEL was developed. Objective: To introduce the process of elaboration of the tool, its applications, potentialities and limits, with its future perspectives. Method: The panel was built from the deterministic relationship of diagnostic and treatment information obtained from the Outpatient Information System, through the Individualized Outpatient Production and the High Complexity Procedure Authorization, in the Hospital Information System and the Cancer Information System, using the National Health Card and the diagnosis of cancer as identification key of the case. Result: The tool has three panels: $1^{\text {st }}$. Panel: Monitoring the initiation of the cancer treatment which excludes non-melanoma and thyroid skin cancers; $2^{\text {nd }}$ Panel: Cases without date of diagnosis by year of treatment, and $3^{\text {rd }}$. Panel: Cases diagnosed with non-melanoma and thyroid skin cancer; and a set of charts and tables with selection filters. Conclusion: OncologyPANEL was available to managers and control agencies on May 15, 2019. It is an agile and manager-accessible tool for monitoring the time of the first cancer treatment. However, the information presented in the OncologyPANEL depend on the registration and the quality of the information of the Health Information Systems from which the data are obtained.

Key words: Health Information Systems; Medical Oncology; Monitoring; Health Information Management; Medical Record Linkage.
\end{abstract}

Resumen

Introducción: La necesidad de controlar el intervalo entre diagnóstico de una neoplasia maligna y el comienzo del tratamiento del cáncer en el Sistema de Salud Pública exigió la construcción de una nueva herramienta. Para este propósito, se desarrolló el PANEL-oncología. Objetivo: Presentar el proceso de elaboración de la herramienta, sus aplicaciones, potencialidades y límites, seńalando perspectivas futuras. Método: El panel se construyó a partir de relación determinista de información de diagnóstico y tratamiento obtenida del Sistema de información para pacientes ambulatorios, a través del Boletín de producción ambulatoria individualizado y Autorización de procedimiento de alta complejidad, Sistema de información hospitalaria y Sistema de información sobre el cáncer, utilizando la Tarjeta nacional de salud y diagnóstico de cáncer como clave de identificación del caso. Resultados: La herramienta tiene tres paneles: 1 - Panel: Monitoreo del inicio del tratamiento del cáncer, que excluye los cánceres de piel no melanoma y de tiroides; 2 - Panel: Casos sin fecha de diagnóstico por año de tratamiento, 3 - Panel: Casos diagnosticados con cáncer de piel no tiroideo y melanoma; y un conjunto de gráficos y tablas con filtros para la selección. Conclusión: PANEL-Oncología se puso a disposición de los gerentes y las agencias de control el 15 de mayo de 2019. Es una herramienta ágil y accesible para los gerentes para monitorear el momento del primer tratamiento contra el cáncer. Sin embargo, la información presentada en el PANEL-Oncología depende del registro y la calidad de información de los Sistemas de la Información de Salud de los cuales consume información. Palabras clave: Sistemas de Información en Salud; Oncología Médica; Monitoreo; Gestión de la Información en Salud; Registro Médico Coordinado.

\footnotetext{
${ }^{1}$ Instituto Nacional de Câncer José Alencar Gomes da Silva (INCA). Rio de Janeiro (RJ), Brasil. Orcid iD: https://orcid.org/0000-0003-2271-746X

${ }^{2}$ INCA. Rio de Janeiro (RJ), Brasil. Orcid iD: https://orcid.org/0000-0002-3075-2591

${ }^{3}$ INCA. Rio de Janeiro (RJ), Brasil. Orcid iD: https://orcid.org/0000-0002-5847-9830

${ }^{4}$ INCA. Rio de Janeiro (RJ), Brasil. Orcid iD: https://orcid.org/0000-0002-4861-2319

${ }^{5}$ INCA. Rio de Janeiro (RJ), Brasil. Orcid iD: https://orcid.org/0000-0002-2472-3444

Endereço para correspondência: Adriana Tavares de Moraes Atty. Rua Marquês de Pombal, 125, 70 andar - Centro. Rio de Janeiro (RJ), Brasil. CEP 22.230-240. E-mail: aatty@inca.gov.br
} 


\section{INTRODUÇÃO}

A organização da rede de atenção à saúde para o controle do câncer exige do gestor um planejamento capaz de atender às demandas de diagnóstico com qualidade $\mathrm{e}$ em tempo oportuno e que garanta o início do tratamento oncológico assim que indicado. A Política Nacional para a Prevençáo e Controle do Câncer $^{1}$ destaca, entre seus princípios e diretrizes, a utilização das bases de dados, informações epidemiológicas e assistenciais disponíveis para o planejamento, monitoramento e avaliaçáo das açôes e serviços. Qualquer iniciativa de detecção precoce que desconsidere esse planejamento não impactará na mortalidade nem na qualidade de vida dos usuários ${ }^{2}$.

$\mathrm{O}$ monitoramento da rede com vistas a identificar possíveis entraves que estejam postergando o início do tratamento e comprometendo o prognóstico exige ferramentas de fácil utilização que auxiliem o gestor nesse processo. Dessa forma, a promulgação da Lei n. ${ }^{\circ} 12.732$, de 22 de novembro de $2012^{3}$, que dispóe sobre o direito de o paciente receber o primeiro tratamento oncológico em até 60 dias a contar da data do diagnóstico, suscitou a necessidade de desenvolver uma ferramenta que permitisse monitorar o tempo dos casos oncológicos tratados no Sistema Único de Saúde (SUS). A Portaria MS/GM n. ${ }^{\circ}$ 876 , de 16 de maio de $2013^{4}$, regulamenta a referida Lei e retira do monitoramento do tempo de tratamento os cânceres não melanóticos de pele dos tipos basocelular e espinocelular e o câncer de tireoide sem fatores clínicos pré-operatórios prognósticos de alto risco.

À época, entendeu-se que essa ferramenta poderia ser uma extensão do Sistema de Informação do Câncer (Siscan), que foi desenvolvido para registrar informaçôes gerenciais referentes aos programas de controle dos cânceres de mama e colo do útero, reunindo o Sistema de Informação do Controle do Câncer do Colo do Útero (Siscolo) e o Sistema de Informaçáo do Câncer de Mama (Sismama) $)^{5}$.

Em 2013, o chamado "módulo tratamento", que previa a inserção de informaçôes sobre o diagnóstico e sobre o primeiro tratamento de cada caso, foi implementado no Siscan. Contudo, após aproximadamente cinco anos de implantação desse módulo, avaliou-se que essa estratégia não foi bem-sucedida, especialmente em virtude da subnotificação de casos, ausência de críticas na entrada de dados e de relatórios que permitissem ao gestor monitorar o tempo.

Considerando a necessidade de reformular a proposta, o Instituto Nacional de Câncer José Alencar Gomes da Silva (INCA), por meio da Divisão de Detecção Precoce, foi convidado, em 2018, a colaborar com uma nova solução que permitisse aos gestores acompanhar o tempo até o tratamento dos casos de câncer em seu território. E, em parceria com o Departamento de Informática do Sistema Único de Saúde (DATASUS), passou-se a desenhar uma nova solução que não representasse um retrabalho para os estabelecimentos de saúde.

Em razão da existência dos Sistemas de Informação em Saúde (SIS) no SUS, já consolidados e de uso rotineiro pelos estabelecimentos de saúde, entendeu-se que a melhor estratégia seria utilizar os dados já disponíveis nesses sistemas para obter as informaçôes necessárias sobre o intervalo entre o diagnóstico de uma neoplasia maligna e o seu primeiro tratamento oncológico no SUS. Assim, a nova ferramenta de gestâo deveria apresentar esse intervalo de tempo, consumindo as informaçóes já enviadas pelos estabelecimentos de saúde nos SIS.

O presente artigo tem como objetivo descrever os princípios e métodos utilizados na construção do PAINEL-Oncologia, bem como discutir suas aplicaçôes, potencialidades e limites, apontando ainda perspectivas futuras.

\section{MÉTODO}

Conforme descrito no Quadro 1, foram utilizados os seguintes SIS-SUS: o Sistema de Informação Ambulatorial $(\mathrm{SIA})^{6}$, por meio do Boletim de Produção Ambulatorial Individualizado (BPA-I) e da Autorizaçáo de Procedimento de Alta Complexidade (Apac); o Sistema de Informação Hospitalar $(\mathrm{SIH})^{7}$; e o Siscan.

Contudo, como os SIS não são interligados, houve a necessidade de estabelecer, a priori, algumas regras e critérios de forma a relacionar as informaçôes sobre diagnóstico e tratamento desses SIS, tais como: definição dos casos de câncer, seleção dos procedimentos com finalidade diagnóstica e de tratamento, e suas respectivas fontes de informação com vistas a obter o intervalo até o primeiro tratamento oncológico.

A proposta baseou-se em um relacionamento determinístico entre os SIS-SUS pela chave identificadora de um caso de neoplasia composta pelo diagnóstico - três primeiros caracteres da Classificaçáo Estatística Internacional de Doenças e Problemas Relacionados à Saúde (CID-10) - e pela identificação do paciente no SUS - Cartão Nacional de Saúde (CNS).

Com a possibilidade de um paciente possuir mais de um CNS, inviabilizando relacionar com precisão as informaçóes de diagnóstico com as informações de tratamento, recorreu-se à base nacional do cartão SUS - CADSUS Web (Cadweb), que agrega todos os possíveis números de CNS de um mesmo paciente em um único CNS: o CNS master ${ }^{8}$. Tal processo foi desenvolvido e implementado pelo DATASUS e incluiu 
Quadro 1. Fontes de dados e critérios de seleção para estruturação do PAINEL-Oncologia

\begin{tabular}{|c|c|c|c|c|}
\hline $\begin{array}{l}\text { Sistema de } \\
\text { Informação }\end{array}$ & Registro & Procedimentos & Finalidade & $\begin{array}{c}\text { Diagnóstico } \\
\text { (CID-10) }\end{array}$ \\
\hline \multirow[b]{3}{*}{ SIA } & \multirow{2}{*}{ BPA-I } & Anatomopatológico & Diagnóstico & \multirow{5}{*}{$\begin{array}{l}\text { C00 ao C97 } \\
\text { D00 ao D09 } \\
\text { D37 ao D48 }\end{array}$} \\
\hline & & Cirurgia & Tratamento & \\
\hline & Apac-Onco & $\begin{array}{l}\text { Quimioterapia, } \\
\text { radioterapia, } \\
\text { hormonioterapia }\end{array}$ & Tratamento & \\
\hline \multirow{2}{*}{$\mathrm{SIH}$} & \multirow{2}{*}{ AlH } & Cirurgia & Tratamento & \\
\hline & & Anatomopatológico & Diagnóstico & \\
\hline Siscan & BPA-I & Anatomopatológico & Diagnóstico & $\begin{array}{c}\text { C50, C53, D05 e } \\
\text { D06 }\end{array}$ \\
\hline
\end{tabular}

etapas de verificação e validação, além de relacionamento probabilístico ${ }^{9,10}$. Portanto, o Cadweb foi a base que possibilitou relacionar os casos diagnosticados com os tratados mediante o cartáo SUS master e as CID informadas nos demais SIS.

O processo de trabalho preservou as informaçōes do local de residência do usuário referentes ao momento em que foi realizado o diagnóstico e o tratamento, para que eventuais atualizaçóes de endereço no Cadweb não implicassem em erros na identificação da origem dos casos de câncer. Destaca-se que os casos que não possuíam CNS master não foram incluídos na ferramenta.

No primeiro momento, definiu-se que o procedimento anatomopatológico seria o único considerado para o diagnóstico, pois aproximadamente $85 \%$ dos casos de câncer foram de diagnóstico histopatológico, conforme verificado na base de dados dos Registros de Câncer de Base Populacional (RCBP), no período de 2010 a $2014^{11}$. Esse procedimento pode ser informado no SIA, por meio do BPA-I; e, no SIH, pela Autorização de Internação Hospitalar (AIH), como procedimento secundário; ou seja, sempre vinculado a um procedimento principal ${ }^{6,7}$.

Para os cânceres de mama e colo do útero, as informações diagnósticas foram obtidas principalmente no Siscan, que gera automaticamente para o SIA os arquivos BPA-I dos procedimentos anatomopatológicos específicos para esses cânceres.

Para essa proposta, foi necessário tornar obrigatório no BPA-I o registro do CNS e CID-10 para o procedimento anatomopatológico não específico dos cânceres do colo do útero e de mama. Essa normatização foi estabelecida pela Portaria SAS n. ${ }^{\circ}$ 643, de 17 de maio de $2018^{12}$, exigindo que constassem no BPA-I, para o procedimento Exame anatomopatológico para congelamento/parafina por peça cirúrgica ou por biópsia (exceto colo uterino e mama), as informaçóes de CNS e CID-10. A Portaria também destaca que a CID-10 a ser utilizada pelo laboratório deverá ser a do resultado do laudo do exame anatomopatológico. Complementarmente, foi publicada a Portaria SAS n. ${ }^{\circ} 202$, de 8 de fevereiro de $2019^{13}$, que qualifica as CID-10 compatíveis com o procedimento anatomopatológico.

Como tratamento, além dos procedimentos de quimioterapia, hormonioterapia e radioterapia informados no SIA pelo módulo "Autorizaçóes de Procedimentos de Alta Complexidade em Oncologia (Apac-Onco)", foram selecionadas no SIH as cirurgias com diagnóstico de câncer informado na AIH; e quatro procedimentos cirúrgicos que são informados no SIA: tratamento cirúrgico de neoplasia de esclera, exérese de tumor de conjuntiva, excisão tipo 1 do colo uterino e excisáo tipo 2 do colo uterino.

Embora a Portaria n. ${ }^{\circ} 876^{4}$, que regulamenta a Lei n. ${ }^{\circ}$ 12.732 , de 22 de novembro de $2012^{3}$, tenha excluído do monitoramento casos de neoplasias de pele e de tireoide, foram recuperados todos os diagnósticos de neoplasia maligna (C00 ao C97), neoplasias in situ (D00 ao D09) e neoplasias de comportamento incerto ou desconhecido (D37 ao D48).

Foram recuperadas as informaçóes diagnósticas desde 2013 para os casos oriundos do Siscan (cânceres do colo do útero e de mama) e, a partir de maio de 2018, para os demais casos informados no SIA. Para os procedimentos recuperados do $\mathrm{SIH}$, a data de internação foi utilizada como proxy da data de início de tratamento.

Uma vez definido o CNS master pelo Cadweb, este foi agregado à CID do diagnóstico e à CID do tratamento nas bases de diagnóstico e de tratamento, compondo uma chave que definiu um caso de câncer (chave CID + CNS) (Figura 1).

Para identificação das datas de diagnóstico e de tratamento, foram aplicadas as seguintes regras às bases de diagnóstico e de tratamento:

- Quando, para uma mesma chave, ou seja, um mesmo caso de câncer, houve mais de uma data de diagnóstico (SIA ou Siscan), foi mantida a mais antiga. 
- Quando, para uma mesma chave, houve mais de uma data de tratamento (SIH, Apac e SIA), foi mantida a mais antiga e posterior à data do diagnóstico (SIA ou Siscan).

- Quando, para uma chave, não foi possível encontrar a data do diagnóstico (SIA ou Siscan) no período estabelecido mas havia na $\mathrm{AIH}$, procedimento secundário, ou na Apac-Onco, na campo data de identificação patológica, essa informação foi utilizada.

- Quando, para uma chave, houve a mesma data de tratamento, tanto na AIH quanto na Apac-Onco, foi recuperada a informação disponível na AIH.

- Quando, para uma mesma chave, houve a mesma data de tratamento, tanto na Apac-Onco de quimioterapia quanto na Apac-Onco de radioterapia, os casos foram reunidos na modalidade terapêutica "quimio+radio", construída para indicar que ambos os tratamentos tiveram a mesma data de início de tratamento.
- Casos com data de tratamento cirúrgico anterior à data do diagnóstico (cirurgia com histopatológico posterior) foram considerados quando a data entre a cirurgia e o resultado anatomopatológico não foi superior a 90 dias. Esse critério de tempo foi estabelecido após consultar especialistas da área de Anatomia Patológica.

Após a aplicação dessas regras, foi construído um banco de dados com informaçóes diagnósticas e outro com informaçóes de tratamento. Por intermédio da chave unívoca (CID + CNS) para cada caso de câncer, foi feito um relacionamento determinístico que reuniu as informaçóes de diagnóstico e tratamento em uma única base de dados.

Os casos com informação de tratamento em que não foi possível identificar a data de diagnóstico, seja no BPA-I ou recuperando na AIH ou Apac-Onco, foram identificados e apresentados em um painel separado por ano de tratamento (Figura 1).
Base de dados de diagnósticos

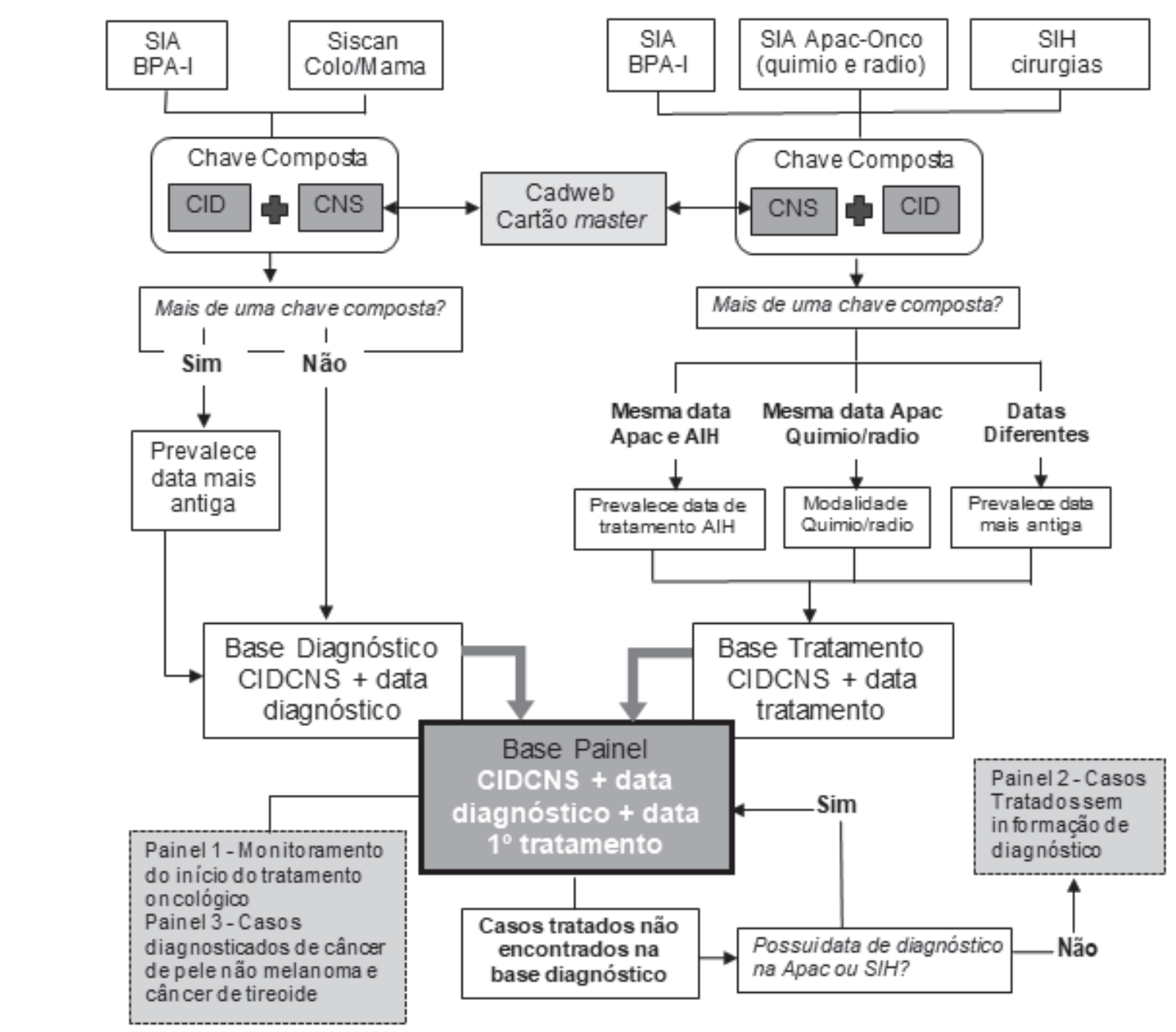

Base de dados de tratamento

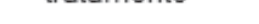

Figura 1. Fluxo para elaboração do PAINEL-Oncologia 
As variáveis constantes no banco final são: a) Estado e município dos estabelecimentos que realizaram o diagnóstico e o tratamento - obtidos pelo Cadastro Nacional de Estabelecimentos de Saúde (CNES), utilizando-se o código CNES; b) CNES diagnóstico e CNES tratamento - código CNES do estabelecimento de saúde; c) Estado e município de residência informados nas bases de dados utilizadas; d) CNS - cartáo master registrado no Cadweb; e) Sexo registrado no Cadweb; f) Idade - diferença entre a data do diagnóstico e a data do nascimento registrada no Cadweb; g) Diagnóstico - CID-10 informado nas bases de dados utilizadas; h) Estadiamento - recuperado da Apac quimioterapia e radioterapia (essa informação não está disponível na AIH, portanto os casos submetidos à cirurgia não têm informação de estadiamento); i) Modalidade terapêutica - cirurgia, quimioterapia, radioterapia e quimio + radio; j) Tempo até o primeiro tratamento - 0 a 30 dias, de 30 a 60 dias, mais de 60 dias, e sem informação de tratamento.

Dada a necessidade de preservar o sigilo das informaçôes disponíveis, limitou-se o acesso pela criação de perfis: Nacional - Ministério da Saúde, Órgãos de controle nacional, Conselho Nacional de Secretários de Saúde (Conass), Conselho Nacional das Secretarias Municipais de Saúde (Conasems); Estadual - Estados, Regionais de Saúde, Órgãos de controle estaduais, Conselho das Secretarias Municipais de Saúde (Cosems); Municipal - Estabelecimento de saúde que realizou o tratamento - Hospital Especializado, Hospital geral, Hospital-Dia.

A liberação de acesso é descentralizada pela ferramenta específica que permite gerenciar o acesso aos usuários.

Com isso, tornou-se possível monitorar o tempo de início de tratamento oncológico por meio de um painel que foi denominado PAINEL-Oncologia, o qual substituiu o chamado "módulo tratamento" que era acoplado ao Siscan.

\section{RESULTADOS}

O PAINEL-Oncologia ${ }^{14}$ foi disponibilizado em 15 de maio de 2019. Não se trata de uma plataforma para inserção de dados. Ele apresenta as informaçóes obtidas dos SIS-SUS distribuídos em tabelas e gráficos disponíveis para download.

A visualizaçáo dos casos identificados depende do perfil de acesso do usuário: o perfil nacional visualiza as informaçóes do país, os perfis estadual e municipal visualizam os dados apenas do seu território e o perfil estabelecimento de saúde dos casos que tratou.

O PAINEL-Oncologia, permite fazer relatórios sobre intervalo entre a data do diagnóstico e a do tratamento considerando a combinação das variáveis: sexo, faixa etária, município e estado de residência, e estabelecimento de saúde que realizou o diagnóstico, estabelecimento de saúde que realizou o tratamento, modalidade terapêutica do primeiro tratamento, estadiamento para os casos tratados com quimioterapia ou radioterapia.

Há ainda a possibilidade de relacionar informaçóes sobre os locais de residência, diagnóstico e tratamento com o tempo para o início do tratamento.

A visualizaçáo das informaçóes no PAINEL-Oncologia é apresentada em três painéis: 1 - Painel: Monitoramento do início do tratamento oncológico; 2 - Painel: Casos sem data de diagnóstico por ano de tratamento e; 3 - Painel: Casos diagnosticados de câncer de pele náo melanoma e câncer de tireoide (Figura 1). Em cada painel, existem já determinados os layouts de tabelas e gráficos que são atualizados conforme a aplicação dos filtros adicionados pelo usuário (Quadro 2).

O PAINEL-Oncologia apresenta o intervalo de tempo entre o diagnóstico e o primeiro tratamento, segundo os intervalos de tempo estabelecidos. Apenas na Tabela 10 do painel 1 é apresentada a data diagnóstica dos casos não tratados e, na Tabela 8 do painel 2, é apresentada a data de tratamento dos casos em que náo foi possível recuperar a data diagnóstica.

$\mathrm{Na}$ data da sua liberação, 15 de maio de 2019, havia 1.170 .063 casos de neoplasias malignas diagnosticados no SUS, distribuídos entre os anos de 2013 a 2019 (Tabela 1).

Entre 2013 e 2017, um terço dos casos diagnosticados foi tratado em até 30 dias, alcançando $36,9 \%$ no ano de 2018. O percentual de casos tratados em até 60 dias foi de aproximadamente $50 \%$ nos anos disponíveis. Nos anos de 2018 e 2019, o percentual de casos que ainda não possuíam informação do primeiro tratamento é elevado (Tabela 1).

No período de 2013 a maio de 2019, para os cânceres mais incidentes no Brasil, observa-se que, com exceção do câncer de pulmão, os casos, em sua maioria, foram tratados após 60 dias da data do diagnóstico (Tabela 2).

A atualização do painel é mensal, havendo alteração nos totais apresentados conforme a inclusáo de novos dados de diagnóstico e de tratamento nos SIS consultados e, conforme a atualização do Cadweb, que permite a incorporação de novos casos ao PAINEL-Oncologia. Destacando-se, portanto, a característica dinâmica dos dados expostos no painel.

Observou-se a ocorrência de idades negativas, que foram tratadas como idades ignoradas. Como as idades foram calculadas pela diferença entre data diagnóstica e data de nascimento, a ocorrência de idades negativas pode ser em decorrência de erro na data de nascimento no Cadweb ou na data diagnóstica. 
Quadro 2. PAINEL-Oncologia: painéis segundo perfil nacional e layouts de tabelas e gráficos

\section{Relatórios dos painéis 1 e 3}

Gráfico 1. Casos de câncer diagnosticados, por ano, segundo tempo até o primeiro tratamento no SUS

Gráfico 2. Número de casos de câncer diagnosticados por ano e faixa etária

Gráfico 3. Número de casos de câncer diagnosticados e tempo até o primeiro tratamento no SUS

Tabela 1. Número de casos de câncer, segundo ano do diagnóstico e tempo até o primeiro tratamento no SUS

Tabela 2. Número de casos de câncer, segundo tempo e tipo de modalidade terapêutica do primeiro tratamento, realizados no SUS

Tabela 3. Número de casos de câncer diagnosticados no SUS, segundo sexo

Tabela 4. Número de casos de câncer, segundo diagnóstico e tempo até o primeiro tratamento no SUS

Tabela 5. Número de casos de câncer, segundo Estado de residência e tempo até o primeiro tratamento no SUS

Tabela 6. Número de casos de câncer, segundo Estado e município de residência e tempo até o primeiro tratamento no SUS

Tabela 7. Número de casos de câncer, segundo unidade de tratamento e tempo até o primeiro tratamento no SUS

Tabela 8. Número de casos de câncer diagnosticados, segundo faixa etária e tempo até o primeiro tratamento no SUS

Tabela 9. Número de casos de câncer, segundo unidade diagnóstica e tempo até o primeiro tratamento no SUS

Tabela 10. Casos de câncer sem informação de tratamento ${ }^{1}$

\section{Relatórios do painel 2}

Gráfico 1. Número de casos de câncer, sem informação de data de diagnóstico, tratados no SUS, por ano e faixa etária

Tabela 1. Número de casos de câncer, sem informação de data de diagnóstico, tratados no SUS, segundo modalidade terapêutica

Tabela 2. Número de casos de câncer, sem informação de data de diagnóstico, tratados no SUS, segundo sexo

Tabela 3. Número de casos de câncer, sem informação de data de diagnóstico, tratados no SUS, segundo diagnóstico principal

Tabela 4. Número de casos de câncer, sem informação de data de diagnóstico, tratados no SUS, segundo Estado de residência

Tabela 5. Número de casos de câncer, sem informação de data de diagnóstico, tratados no SUS, segundo Estado e município de residência

Tabela 6. Número de casos de câncer, sem informação de data de diagnóstico, tratados no SUS, segundo unidade de tratamento e modalidade terapêutica

Tabela 7. Número de casos de câncer, sem informação de data de diagnóstico, tratados no SUS, segundo faixa etária

Tabela 8. Lista de pacientes sem data de diagnóstico por ano de tratamento

Nota: ${ }^{1}$ Apresentado apenas no painel 1 .

Fonte: PAINEL-Oncologia ${ }^{14}$.

Para um número muito reduzido de casos $(0,04 \%$ em agosto de 2019), observaram-se datas de tratamento futuras à data corrente do painel, indicando problema na crítica das datas dos sistemas de informação utilizados.

\section{DISCUSSÃO}

Alguns estudos têm sido realizados em nível local ou nacional, para a identificação do tempo transcorrido entre o diagnóstico e o primeiro tratamento, assim como os fatores associados aos possíveis atrasos ${ }^{15-17}$. Contudo, tais estudos são elaborados por tipos específicos de câncer, tendo importantes restriçóes do seu escopo quanto ao período e local de origem dos dados, e utilizam coleta de dados em prontuários, entrevistas, ou dados secundários retrospectivos, demonstrando a complexidade na obtenção dessas informações e a inovação trazida pelo PAINEL-Oncologia.

Comparado ao chamado "módulo tratamento" do Siscan, o PAINEL-Oncologia trouxe inúmeras vantagens, entre as quais se destacam: (a) o aumento muito expressivo do número de casos captados; (b) o aumento da eficiência 
Tabela 1. Distribuição dos casos de neoplasia* diagnosticados por ano do diagnóstico, segundo intervalo de tempo até início do tratamento oncológico no SUS. Brasil, 2013-2019

\begin{tabular}{c|c|c|c|c|c}
\hline \multirow{2}{*}{ Ano } & 0 a 30 dias & $\mathbf{3 1}$ a $\mathbf{6 0}$ dias & Mais de $\mathbf{6 0}$ dias & $\begin{array}{c}\text { Sem informação } \\
\text { de tratamento }\end{array}$ & Total \\
\cline { 2 - 6 } & $\%(\mathbf{N})$ & $\%(\mathbf{N})$ & $\%(\mathbf{N})$ & $\%(\mathbf{N})$ & $\mathbf{N}$ \\
\hline 2013 & $31,2(49.292)$ & $19,4(30.559)$ & $49,4(77.975)$ & $0,1(92)$ & 157.918 \\
2014 & $29,7(48.813)$ & $19,0(31.184)$ & $49,4(81.184)$ & $1,9(3.196)$ & 164.377 \\
2015 & $29,3(48.877)$ & $19,4(32.374)$ & $48,7(81.316)$ & $2,6(4.359)$ & 166.926 \\
2016 & $29,8(51.222)$ & $19,8(34.074)$ & $47,4(81.607)$ & $3,0(5.170)$ & 172.073 \\
2017 & $29,9(52.833)$ & $20,1(35.484)$ & $46,5(82.183)$ & $3,6(6.277)$ & 176.777 \\
2018 & $36,9(103.494)$ & $12,9(36.067)$ & $24,3(67.991)$ & $25,9(72.610)$ & 280.162 \\
2019 & $43,8(22.720)$ & $6,5(3.392)$ & $1,3(649)$ & $48,4(25.069)$ & 51.830 \\
Total & $32,2(377.251)$ & $17,4(203.134)$ & $40,4(472.905)$ & $10,0(116.773)$ & 1.170 .063 \\
\hline
\end{tabular}

Nota: *Excluídos os casos de câncer de pele e tireoide.

Fonte: PAINEL-Oncologia ${ }^{14}$.

Tabela 2. Distribuição dos casos diagnosticados por tipo de câncer selecionado, segundo o intervalo até o início do tratamento oncológico no SUS. Brasil, 2013-2019

\begin{tabular}{l|c|c|c|c}
\hline Cânceres mais incidentes & $\begin{array}{c}\text { Até } 30 \text { dias } \\
\%(\mathbf{n})\end{array}$ & $\begin{array}{c}31-60 \text { dias } \\
\%(\mathbf{n})\end{array}$ & $\begin{array}{c}\text { Mais de } 60 \\
\%(\mathbf{n})\end{array}$ & $\begin{array}{c}\text { Sem informação } \\
\text { de tratamento } \\
\%(\mathbf{n})\end{array}$ \\
\hline $\begin{array}{l}\text { Neoplasia maligna da mama } \\
\text { (C50) }\end{array}$ & $27,30(62.712)$ & $19,77(45.426)$ & $48,86(112.259)$ & $4,07(9.352)$ \\
$\begin{array}{l}\text { Neoplasia maligna da } \\
\text { próstata (C61) }\end{array}$ & $19,51(29.850)$ & $13,31(20.358)$ & $61,21(93.652)$ & $5,97(9.130)$ \\
$\begin{array}{l}\text { Neoplasia maligna do cólon } \\
\text { (C18) }\end{array}$ & $41,51(25.855)$ & $20,13(12.539)$ & $32,58(20.293)$ & $5,78(3.603)$ \\
$\begin{array}{l}\text { Neoplasia maligna dos } \\
\text { brônquios e dos pulmões }\end{array}$ & $43,36(24.351)$ & $25,5914.369)$ & $26,55(14.909)$ & $4,50(2.528)$ \\
$\begin{array}{l}\text { (C33 e C34) } \\
\text { Neoplasia maligna do colo } \\
\text { do útero (C53) }\end{array}$ & $23,35(16.619)$ & $19,68(14.006)$ & $48,82(34.751)$ & $8,15(5.799)$ \\
$\begin{array}{l}\text { Neoplasia maligna do reto } \\
\text { (C20) }\end{array}$ & $23,13(9.387)$ & $24,35(9.880)$ & $48,02(19.489)$ & $4,50(1.825)$ \\
\hline
\end{tabular}

Fonte: PAINEL-Oncologia ${ }^{14}$.

pela ausência de retrabalho por usar dados de outros SIS, não sendo necessário implantar um novo sistema ou treinar funcionários para a obtenção e inserção de dados; (c) a melhoria da qualidade dos dados em função da ausência de crítica na entrada de dados da versão anterior e por usar dados ligados ao faturamento. Dessa forma, o painel torna-se uma ferramenta mais ágil e acessível ao gestor para o monitoramento do tempo do primeiro tratamento do câncer, utilizando as informaçóes já registradas nos SIS-SUS.

As informações apresentadas no PAINEL-Oncologia dependem do registro e da qualidade das informaçóes dos
SIS, dos quais ele consome informaçôes: SIA (BPA-I, Apac-Onco), SIH, Siscan, CNES e Cadweb. Com atualização mensal, as proporçóes de casos tratados e não tratados podem ser alteradas pela captação de novas informaçôes. Em função da pressão legal pelo monitoramento do tempo entre o diagnóstico e o primeiro tratamento, é provável que a utilização da ferramenta fomentará a qualificação dos dados dos SIS que os alimentam, resultando na melhoria contínua das informaçóes para o próprio painel.

Face ao insucesso da implantação da solução anterior (o chamado "módulo tratamento"), outra vantagem do PAINEL-Oncologia é a possibilidade 
de captar informaçóes de períodos anteriores ao seu lançamento em maio de 2019. Isso ocorre em virtude do uso de informaçóes de SIS já existentes e disponíveis para acesso.

Portanto, no caso dos cânceres de mama e do colo do útero, o painel consegue apresentar dados desde $2013 \mathrm{em}$ virtude das bases de dados oriundos do sistema criado para as ações de detecção precoce desses cânceres, o Siscan, o qual já tinha como campos obrigatórios a informação do CNS e do CID. Já para os outros tipos de câncer, de forma geral, as informaçóes ficaram disponíveis a partir de maio de 2018, um ano antes do lançamento do painel, em função da obrigatoriedade dos campos CNS e CID-10 para os procedimentos anatomopatológicos ${ }^{12}$.

Cabe destacar que a utilização da informação diagnóstica registrada no SIH e Apac-Onco para os casos tratados e sem a recuperação da informação diagnóstica no SIA e Siscan possibilitou obter até mesmo informaçôes anteriores a maio de 2018. O que permitiu captar informaçóes referentes a qualquer procedimento diagnóstico, não apenas de exames anatomopatológicos, uma vez que na Apac-Onco a informação do campo diagnóstico considera a data do diagnóstico de neoplasia independente do procedimento diagnóstico realizado.

O PAINEL-Oncologia é uma importante ferramenta de gestão, pois disponibiliza informaçôes para além do previsto na Lei n. ${ }^{\circ} 12.732^{3}$, de 22 de novembro de 2012. O PAINEL-Oncologia oferece ao gestor a possibilidade de identificar possíveis entraves que estejam postergando o início do tratamento e comprometendo o prognóstico. A divisão da visualização do painel em três painéis permite monitorar os casos diagnosticados fora da rede pública de saúde, mas tratados no SUS, e ainda os casos em que não foi possível recuperar a data de diagnóstico. Assim como apresenta os casos de câncer de pele não melanoma e câncer de tireoide, para uma análise em separado, não interferindo no cálculo geral do tempo de tratamento dos demais cânceres.

Considerando a importância da temática e do acesso à informação, foi disponibilizado o Tabnet do painel (http:// tabnet.datasus.gov.br/cgi/dhdat.exe?PAINEL_ONCO/ PAINEL_ONCOLOGIABR.def), de acesso livre e irrestrito, que permite o acompanhamento do intervalo entre o diagnóstico e o início do tratamento oncológico de forma anonimizada.

Vale ressaltar que as informaçóes do PAINEL-Oncologia não se referem à incidência de câncer no país, pois limitam-se aos casos cujo dados estáo disponíveis nas bases de dados utilizadas no SUS, sem identificação de casos novos. Importante ainda destacar que o painel não substitui as informaçôes disponibilizadas nos Registros Hospitalares de Câncer (RHC) ou nos RCBP.
No momento, o fato de nem todos os CNS apresentarem um cartão master $^{8}$ reflete uma limitação, gerando perda de casos no painel, mas impulsiona que essa questáo seja abordada pelos gestores mais tempestivamente.

Os casos anteriores à Portaria SAS n. ${ }^{\circ} 643^{12}$ - cuja informação diagnóstica não foi recuperada -, os casos diagnosticados fora do SUS, os diagnosticados por procedimento náo anatomopatológico e que tenham tido a cirurgia como primeiro tratamento oncológico compuseram o grupo de casos sem informação diagnóstica. Isso porque a AIH náo contempla a informação de data diagnóstica.

Já para alguns casos submetidos à radioterapia ou à quimioterapia, como primeiro tratamento, independentemente do tipo de procedimento diagnóstico e de ter sido realizado no SUS ou não, foi possível recuperar essa data, pois, nesses sistemas, há um campo para registro dessa informação. Contudo, cabe destacar que a ausência efetiva de críticas no preenchimento da Apac inviabilizou a recuperação da data diagnóstica para a totalidade desses casos.

Acrescentam-se, aos casos sem informação diagnóstica, os cânceres do colo do útero e mama provenientes do Siscolo e Sismama, já que esses sistemas não têm obrigatoriedade de registro do cartão SUS.

A identificação de casos com data de tratamento futura ocasionou a discussão com os gestores desses sistemas e o encaminhamento de inserir a crítica nesses campos no recebimento do arquivo do SIA e da Apac.

A impossibilidade de identificar lateralidade do câncer de mama faz com que casos diferentes sejam considerados um único caso para o CNS.

A existência de múltiplos fragmentos anatomopatológicos com diagnósticos diferentes compondo chaves distintas, ou seja, CID diferente para um mesmo CNS, pode ocasionar falta de correspondência no tratamento para cada um desses diagnósticos.

Ressalta-se ainda que a não implantação do Siscan em todo território nacional permite a utilizaçáo do Siscolo e do Sismama por alguns laboratórios, com perda desses exames histopatológicos.

O PAINEL-Oncologia apresenta majoritariamente informação diagnóstica dos exames anatomopatológicos e estima-se que esses dados correspondem a aproximadamente $85 \%$ dos casos de câncer diagnosticados no SUS ${ }^{11}$. A título de comparação, esse procedimento correspondeu a cerca de 70\% dos cânceres diagnosticados nos Estados Unidos, segundo o National Cancer Database ${ }^{18}$. Contudo, já está em discussão, como melhoria do painel, a inclusão, em um primeiro momento, do procedimento citopatológico, considerado importante no diagnóstico do câncer de pulmão ${ }^{19}$. 


\section{CONCLUSÃO}

O PAINEL-Oncologia permite ao gestor acompanhar os casos de neoplasias de seus residentes e os casos diagnosticados ou tratados em estabelecimentos públicos ou credenciados ao SUS localizados em seu território, e foi concebido com um método que trouxe diversas vantagens com relação à abordagem adotada anteriormente no país.

Entende-se que o PAINEL-Oncologia é uma importante ferramenta de gestão que permitirá ao gestor discutir e reorientar a organização de sua rede de forma a prover melhor acesso ao tratamento.

\section{CONTRIBUIÇÕES}

Adriana Tavares de Moraes Atty e Jeane Glaucia Tomazelli contribuíram na concepção e delineamento do artigo; na análise, interpretação dos dados e redação do texto; e na revisão crítica do conteúdo intelectual do manuscrito. Beatriz Cordeiro Jardim, Maria Beatriz Kneipp Dias e Arn Migowski contribuíram na análise, interpretação dos dados e redação do texto; e na revisão crítica do conteúdo intelectual do manuscrito. Todos os autores aprovaram a versão final a ser publicada.

\section{AGRADECIMENTOS}

À Ivanir Martins de Oliveira pela contribuição na definição do tempo diagnóstico posterior ao tratamento para os casos tratados cirurgicamente. À Teresa Cravo, à Marise Rebelo e à Ana Lúcia Eisenberg pela contribuição na discussão da restrição das CID compatíveis com o procedimento diagnóstico. À Caroline Madalena Ribeiro pela contribuição do desenho da proposta inicial do PAINEL-Oncologia. Aos técnicos do DATASUS, Alzira Falcáo, Rodrigo Saar, Eduardo de Paula e Fabrício Pires, pela participação na efetiva construção do PAINELOncologia, aplicando as regras negociais às bases de dados dos SIS utilizados e pela ajuda na compreensão das bases primárias, fontes do PAINEL-Oncologia.

\section{DECLARAÇÃO DE CONFLITO DE INTERESSES}

Nada a declarar.

\section{FONTES DE FINANCIAMENTO}

Não há.

\section{REFERÊNCIAS}

1. Ministério da Saúde (BR), Gabinete do Ministro. Portaria $\mathrm{n}^{\circ}$ 874, de 16 de maio de 2013. Institui a Política
Nacional para a Prevenção e Controle do Câncer na Rede de Atenção à Saúde das Pessoas com Doenças Crônicas no âmbito do Sistema Único de Saúde (SUS). Diário Oficial da União, Brasília, DF; 2013 maio 17. Seção I, p. 129.

2. World Health Organization. Guide to early cancer diagnosis [Internet]. Geneve: WHO; 2017 [cited 2019 Sep 26]. Available from: http://apps.who.int/iris/bitstre am/10665/254500/1/9789241511940-eng.pdf

3. Presidência da República (BR). Lei no 12.732 , de 22 de novembro de 2012 [Internet]. [acesso 2020 jan 06]. Disponível em: http://www.planalto.gov.br/ CCIVIL_03/_Ato2011-2014/2012/Lei/L12732.htm

4. Ministério da Saúde (BR), Gabinete do Ministro. Portaria $n^{\circ} 876$, de 16 de maio de 2013. Dispóe sobre a aplicaçáo da Lei $n^{\circ} 12.732$, de 22 de novembro de 2012, que versa a respeito do primeiro tratamento do paciente com neoplasia maligna comprovada, no âmbito do Sistema Único de Saúde (SUS). Diário Oficial da União, Brasília, DF; 2013 maio 17. Seção I, p. 135.

5. Instituto Nacional de Câncer José Alencar Gomes da Silva. Sistemas de informação do controle do câncer de mama (SISMAMA) e do colo do útero (SISCOLO): manual gerencial. Rio de Janeiro: INCA; 2011.

6. Ministério da Saúde (BR). Manual técnico operacional SIA/SUS: sistema de informaçôes ambulatoriais: aplicativos de captação da produçấo ambulatorial APAC Magnético - BPA Magnético - VERSIA - DE-PARA - FPO Magnético. Brasília, DF: Ministério da Saúde; 2009.

7. Ministério da Saúde (BR). SIH: sistema de informaçôes hospitalares: manual técnico operacional do sistema. Brasília, DF: Ministério da Saúde; 2017.

8. Ministério da Saúde (BR), Secretaria de Atenção à Saúde, Departamento de Regulação, Avaliaçấo e Controle. Sistemas de informação da atenção à saúde: contextos históricos, avanços e perspectivas no SUS. Brasília, DF: Cidade Gráfica e Editora; 2015. Capítulo 1, CADSUS cadastro nacional de usuários do SUS: evolução histórica e conceitual; p. 23-41.

9. Ministério da Saúde (BR), Gabinete do Ministro. Portaria no 940, de 28 de abril de 2011. Regulamenta o Sistema Cartão Nacional de Saúde (Sistema Cartão). Diário Oficial da União, Brasília, DF; 2011 maio 2. Seção I, p. 58.

10. Ministério da Saúde (BR), Secretaria de Atenção à Saúde. Portaria $\mathrm{n}^{\circ} 174$, de 14 de maio de 2004. Diário Oficial da Uniáo, Brasília, DF; 2004 maio 17. Seção I, p. 62.

11. Instituto Nacional de Câncer José Alencar Gomes da Silva. Registro de câncer de base populacional [Internet]. Rio de Janeiro: INCA; c1996-2018. [acesso 2020 jan 06]. Disponível em: https://www.inca.gov.br/ BasePopIncidencias/Home.action

12. Ministério da Saúde (BR), Secretaria de Atenção à Saúde. Portaria no 643, de 17 maio de 2018. Altera 
atributos do procedimento da Tabela de Procedimentos, Medicamentos, Órteses/Próteses e Materiais Especiais do SUS. Diário Oficial da União, Brasília, DF; 2018 maio 21. Seção I, p. 71.

13. Ministério da Saúde (BR), Secretaria de Atenção à Saúde. Portaria no 202, de 8 fevereiro de 2019. Compatibiliza códigos da CID-10 com procedimento na Tabela de Procedimentos, Medicamentos, Órteses, Próteses e Materiais Especiais do Sistema Único de Saúde/SUS. Diário Oficial da Uniáo, Brasília, DF; 2019 fev 15. Seção I, p. 54.

14. Ministério da Saúde (BR), Departamento de Informática do SUS. PAINEL-Oncologia. Brasília, DF: DATASUS; 2019. [acesso 2019 dez 22]. Disponível em: https:// painel-oncologia-kb.saude.gov.br/

15. Medeiros GC, Bergmann A, Aguiar SS, et al. Análise dos determinantes que influenciam o tempo para o início do tratamento de mulheres com câncer de mama no Brasil. Cad Saúde Pública. 2015;31(6):1269-82. doi: https:// doi.org/10.1590/0102-311X00048514

16. Carvalho PG, O’Dwer G, Rodrigues NCP. Trajetórias assistenciais de mulheres entre diagnóstico e início de tratamento do câncer de colo uterino. Saúde Debate. 2018;42(118):687-701. doi: https://doi. org/10.1590/0103-1104201811812

17. Asssenço KC, Kluthcovsky ACGC, Mansani FP. Atraso no diagnóstico e tratamento de pacientes com câncer de colo de útero atendidas pelo Sistema Único de Saúde em um centro de referência do Sul do Brasil. O Mundo Saúde. 2017;41(4):692-702. doi: https://doi. org/10.15343/0104-7809.20174104692702

18. American College of Surgeons. National Cancer Database [Internet]. Chicago: American College of Surgeons. C1996-2020. [cited 2019 Aug 28]. Available from: https://www.facs.org/quality-programs/cancer/ncdb

19. Rabahi MF, Ferreira AA, Reciputti BP, et al. Achados de fibrobroncoscopia em pacientes com diagnóstico de neoplasia pulmonar. J Bras Pneumol. 2012;38(4):445-51. doi: https://doi.org/10.1590/ S1806-37132012000400006 